\title{
SUMER Observations of the Solar Transition Region: Spatial and Temporal Behaviour
}

\author{
L. Teriaca and J. G. Doyle \\ Armagh Observatory, Armagh BT61 9DG, Northern Ireland \\ D. Banerjee \\ Centre for Plasma Astrophysics, Katholieke Universiteit Leuven, \\ Celestijnenlaan 200B, 3001 Heverlee, Belgium
}

\begin{abstract}
We examine the spatial and temporal behaviour of the quiet Sun transition region (TR) using high resolution SUMER observations in O vi $1032 \AA$. A detailed study of raster images allows us to differentiate the network and internetwork through Doppler shift and line width measurements. The oscillatory nature of the TR is investigated using wavelet analysis over a series of spectra obtained with high temporal cadence.
\end{abstract}

\section{Introduction}

The network is the dominant component of the solar TR, contributing, on O VI, to the $70 \%$ of the total TR emission (Reeves 1976; Gallagher et al. 1998). It is present from the chromosphere up to the low corona, reaching the maximum contrast in the mid TR around $T=2.510^{5} \mathrm{~K}$ (Gallagher et al. 1998). Evidence for the presence of small arcsecond and sub-arcsecond loops within the network has been presented (Dowdy 1993). More recently, small scale loop-like structures extending inside the supergranulation cells, has been found particularly at the boundaries of the supergranulation network (Warren 2000). Many of these structures are characterized by width equal to the spatial resolution and length from few to $\sim 30$ arcseconds (Warren 2000; Feldman et al. 1999).

\section{Observations \& Data reduction}

The observations were obtained in April '96 and consist of four successive raster scans of the same $120 \times 100 \operatorname{arcsec}^{2}$ region followed by a temporal series of spectral images centered at $\lambda 1032 \AA$. Each spectrum was obtained exposing for 6 seconds the central part of detector A through the $1^{\prime \prime} \times 120^{\prime \prime}$ slit. In order to obtain reliable measurements of line position and width across the raster, we binned over groups of four pixels obtaining a final resolution of $2.26 \times 2 \operatorname{arcsec}^{2}$ for a total of 2650 spectra analyzed in each raster scan. Line intensity analysis for the raster images has been carried out using the full resolution rasters. The temporal series consist of 1048 slit images exposed for 6 seconds using the $1^{\prime \prime} \times 120^{\prime \prime}$ slit on detector $\mathrm{A}$. In order to increase the $\mathrm{S} / \mathrm{N}$ ratio, temporal data were binned 

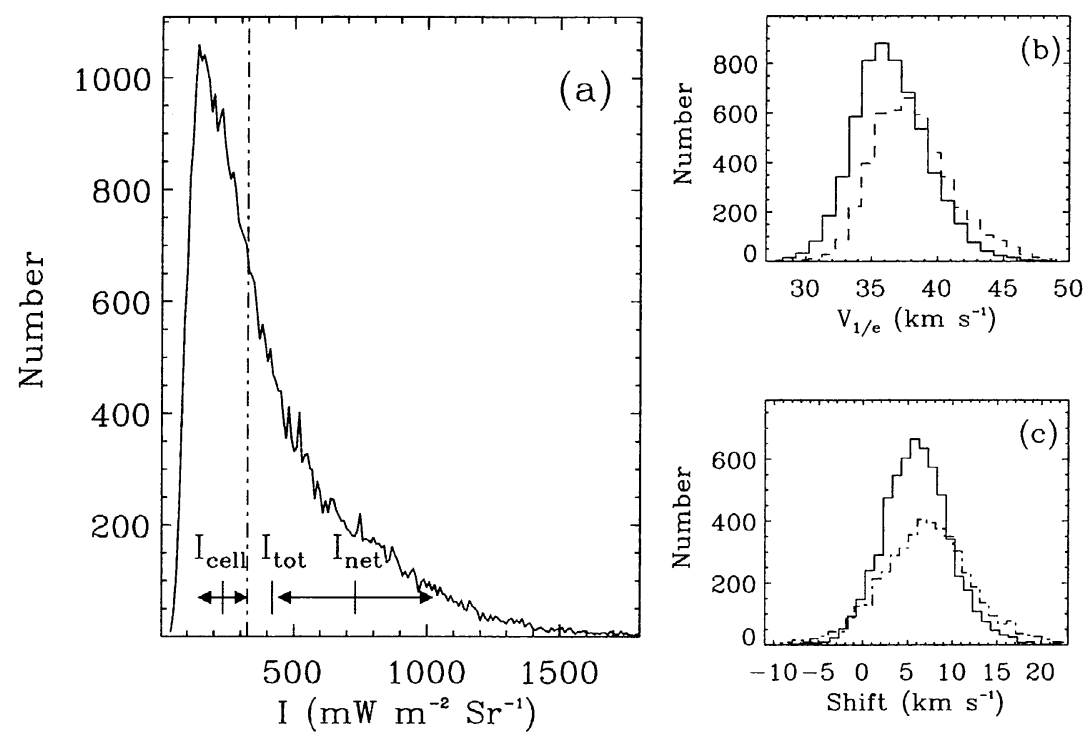

Figure 1. (a) Intensity distribution (histogram) of the four full resolution images. $I_{\text {tot }}$ is the average intensity. $I_{\text {cell }}$ and $I_{n e t}$ the average intensities for points with $I<I_{\text {tot }}$ and $I>I_{\text {tot }}$, respectively. Standard deviations for $I_{\text {cell }}$ and $I_{n e t}$ are also reported. The vertical dot-dashed line indicates the position $I_{\text {cell }}+\sigma_{\text {cell }}$ assumed as separating cell centers from network regions. $\mathrm{V}_{1 / e}(b)$ and Doppler shift (c) distributions for cell centers (solid line) and network regions dashed line.

over 5 pixels along the slit. Data were reduced using various IDL routines from within the SUMER software tree.

\section{Data analysis}

Using the full resolution intensity images we obtained a precise intensity distribution. After defining the total average intensity as $I_{t o t}$, we calculate the mean and the standard deviation for all points with intensity below $\left(I_{\text {cell }} \& \sigma_{\text {cell }}\right)$ and above $\left(I_{n e t} \& \sigma_{n e t}\right) I_{\text {tot }}$. Following Reeves (1976) we define $I_{\text {cell }}+\sigma_{\text {cell }}$ as the intensity value dividing cell centers from network regions (see Fig. 1). Using the above value we generate the position and width distributions in network and internetwork for the binned images. For the temporal series, we perform a wavelet analysis of the high cadence spectra of the 'quiet Sun' obtained in the O VI $1032 \AA$ line, stressing differences between network and internetwork regions, finding that rapid changes in all line parameters are found in both network and internetwork regions. Even if velocity and intensity fluctuations are generally larger in the network areas (with increases in intensity up to a factor 4), the internetwork shows a clear signature of velocity fluctuations up to $6 \mathrm{mHz}$ $(180 \mathrm{~s})$ that are not seen in the network. 


\section{Observational results}

Differences in the width and position distributions of network and cell centers are clearly seen (Fig. 1b \& 1c), with a higher mean value for the distributions relative to the network. The width (position) distribution for the network is well approximated by a single Gaussian centered at $\mathrm{V}_{1 / e}=37.6 \mathrm{~km} \mathrm{~s}^{-1}(\mathrm{~V}=7.1 \mathrm{~km}$ $\left.\mathrm{s}^{-1}\right)$ and having a FWHM of $6.2 \mathrm{~km} \mathrm{~s}^{-1}\left(10.8 \mathrm{~km} \mathrm{~s}^{-1}\right)$. The width (position) distribution for the internetwork is well approximated by a single Gaussian centered at $\mathrm{V}_{1 / e}=36.2 \mathrm{~km} \mathrm{~s}^{-1}\left(\mathrm{~V}=5.9 \mathrm{~km} \mathrm{~s}^{-1}\right)$ and a FWHM of $6.2 \mathrm{~km} \mathrm{~s}^{-1}(8.2$ $\mathrm{km} \mathrm{s}^{-1}$ ). In all the temporal series an oscillatory nature of the line parameters is observed. A coherent oscillatory pattern is clearly visible in the "shift" time slice, with a spatial extension along the slit of $\sim 15^{\prime \prime}$ and lasting more than $500 \mathrm{~s}$. In general, velocity oscillations in the internetwork are more evident than in the network at higher frequencies (up to $6 \mathrm{mHz}$ ). For chromospheric lines, Doyle et al. (1999) find little differences between network and internetwork regions in both the intensity and the velocity power spectrum. A closer examination of the intensity, position and width time slices shows that the TR is inhomogeneous and with dynamic features that are episodic in nature. This makes the wavelet analysis (and its capacity to extract frequency information localized in time) a very powerful tool in the study of the upper solar atmosphere.

\section{Conclusions}

The network is characterized by higher mean values of the distributions of shift and width. The dominant power in the internetwork lies between $1-3 \mathrm{mHz}$. We do not see the dominant power around $5.5 \mathrm{mHz}(3 \mathrm{~min})$, as it is predicted by numerical simulations (Carlsson et al. 1997). We notice weak and short-lived power around $5.5 \mathrm{mHz}$. Intensity and velocity show significant power in 1-3 $\mathrm{mHz}$ range. In the network we find more oscillation in velocity than in intensity. The dominant power is around $2 \mathrm{mHz}$. When we have more counts we see a strong power in the phase plot, suggesting that whatever causes these oscillations, it switches on and off. The wavelet spectra imply that these oscillations come in wave packets lasting roughly $500-1000 \mathrm{~s}$ with spatial scale of $\sim 5^{\prime \prime}$.

Acknowledgments. Research at Armagh Observatory is grant-aided by the N. Ireland Dept. of Culture, Arts and Leisure. This work was supported by PPARC grant PPA/G/S/1999/00055.

\section{References}

Carlsson M., Judge P. J. \& Wilhelm K., 1997, ApJ 486, L63

Doyle J.G., van den Oord G.H.J., O'Shea E., Banerjee D., 1999. A\&A 347, 335

Dowdy J.F.Jr., 1993, ApJ 411, 406

Feldman U., Widing K.G., Warren H.P., 1999, ApJ 522, 1133

Gallagher P.T., Phillips K.J.H., et al., 1998, A\&A 335, 733

Reeves E.M., 1976, Solar Phys 46, 53

Warren H.P., Winebarger A.R., 2000, ApJ L 535, L63 